\title{
Vascular access for hemodialysis: current perspectives
}

This article was published in the following Dove Press journal: International Journal of Nephrology and Renovascular Disease 8 July 2014

Number of times this article has been viewed

\author{
Domenico Santoro' \\ Filippo Benedetto 2 \\ Placido Mondello ${ }^{3}$ \\ Narayana Pipitò ${ }^{2}$ \\ David Barillà ${ }^{2}$ \\ Francesco Spinelli \\ Carlo Alberto Ricciardi' \\ Valeria Cernaro' \\ Michele Buemi' \\ 'Department of Clinical and \\ Experimental Medicine, Unit \\ of Nephrology, ${ }^{2}$ Unit of Vascular \\ Surgery, ${ }^{3}$ Unit of Infectious Disease, \\ University of Messina, Italy
}

\begin{abstract}
A well-functioning vascular access (VA) is a mainstay to perform an efficient hemodialysis (HD) procedure. There are three main types of access: native arteriovenous fistula (AVF), arteriovenous graft, and central venous catheter (CVC). AVF, described by Brescia and Cimino, remains the first choice for chronic HD. It is the best access for longevity and has the lowest association with morbidity and mortality, and for this reason AVF use is strongly recommended by guidelines from different countries. Once autogenous options have been exhausted, prosthetic fistulae become the second option of maintenance HD access alternatives. CVCs have become an important adjunct in maintaining patients on HD. The preferable locations for insertion are the internal jugular and femoral veins. The subclavian vein is considered the third choice because of the high risk of thrombosis. Complications associated with CVC insertion range from 5\% to 19\%. Since an increasing number of patients have implanted pacemakers and defibrillators, usually inserted via the subclavian vein and superior vena cava into the right heart, a careful assessment of risk and benefits should be taken. Infection is responsible for the removal of about $30 \%-60 \%$ of HD CVCs, and hospitalization rates are higher among patients with CVCs than among AVF ones. Proper VA maintenance requires integration of different professionals to create a VA team. This team should include a nephrologist, radiologist, vascular surgeon, infectious disease consultant, and members of the dialysis staff. They should provide their experience in order to give the best options to uremic patients and the best care for their VA.
\end{abstract}

Keywords: arteriovenous fistula, prosthetic grafts, central venous catheter, infection

\section{Introduction}

In the past, one of the major problems and causes of failure in hemodialysis (HD) was represented by the lack of good vascular access (VA). After the introduction of the Cimino-Brescia fistula, in the last few decades, the advent of prosthetic arteriovenous graft (AVG) and central venous catheters (CVCs) has given physicians the opportunity to choose the most appropriate VA for HD patients. However, the native arteriovenous fistula (AVF) remains the first choice for VA, especially because of the infectious and thrombotic complications more frequently associated with AVGs and CVCs. ${ }^{1}$ Due to improved HD technique and a better treatment of comorbidity, dialysis patients now have a higher life expectancy. Aging of HD patients requires an improvement in performing VA so that it is able to last decades.

Epidemiological data on VA use in incident and prevalent end-stage renal disease patients across countries have shown a considerable variation in patient VA preference. In particular, patient preference for a catheter varies across countries, with preference ranging from $1 \%$ of HD patients in Japan and $18 \%$ in the United States, to $42 \%$ and $44 \%$ in Belgium and Canada. ${ }^{2}$

\footnotetext{
Correspondence: Domenico Santoro Division of Nephrology and Dialysis, Department of Clinical and Experimental Medicine, University of Messina, AOU G. Martino PAD C; Via Consolare Valeria, 98100 Messina, Italy Tel +39090 22I 2396 $\mathrm{Fax}+390902212331$

Email dsantoro@unime.it
} 


\section{Arteriovenous fistula (AVF)}

The AVF needs to be planned at least one or two months before starting HD, a time required for the proper maturation of the VA. A correct flow-chart should include a preoperative phase, an operative phase, and a postoperative one.

Clinical and instrumental evaluation is necessary to decide the type of VA, the technical approach, and the correct follow-up to handle complications as early as possible. To preserve the vascular system, it is important to avoid blood withdrawals or intravenous infusions from the arm and forearm, and to use the veins of the hands for these purposes.

The preoperative phase of AVF includes accurate collection of medical history, physical examination, and instrumental evaluation. ${ }^{3}$ Anamnestic collection should investigate about heart diseases, to assess any alteration in cardiac output. Indeed, as a consequence of AVF, there may be changes in blood flow, pulmonary pressure, and cardiac output, especially when the blood flow of the AVF is greater than $2,000 \mathrm{~mL} / \mathrm{min} .{ }^{4}$ Previous arterial and/or venous catheterization needs to be investigated for the high risk of central vein stenosis with consequent reduced venous output of the future VA. It is important to identify the dominant limb in order to avoid a limitation of the patient's quality of life.

The physical examination is aimed to investigate arterial and venous system functioning and therefore exclude the presence of any edema, surgical scars, radial, ulnar and brachial pulses, and superficial venous circles. The Allen test should be performed to evaluate an abnormal vascularization of the palmar arch.

The gold standard to decide on the type and location of VA is the duplex ultrasound scan. It allows the assessment of the arterial and venous diameters; a vein diameter $>2 \mathrm{~mm}$ and an artery diameter $>1.6 \mathrm{~mm}$ are considered adequate. These two parameters are predictive of AVF maturation. ${ }^{5}$

According to the guidelines of the National Kidney Foundation (NKF-K/DOQI), ${ }^{6}$ the site order for the surgical intervention of AVF for HD is the following: forearm (radio-cephalic or distal AVF), elbow (brachio-cephalic or proximal AVF), arm (brachial-basilic AVF with transposition or proximal AVF).

The AVF directly on the wrist is considered the gold standard for VA. It is relatively simple to create, and since there is a low incidence of complications, the long-term patency rates are excellent and do not preclude the possibility of future access. ${ }^{1}$ Different types of arteriovenous anastomoses are possible: side-to-end of the vein on the artery, laterolateral, terminalized side-to-side, side-to-end of the artery on the vein, and end-to-end (Figure 1). The most common is the anastomosis of the vein side-to-end of the artery.

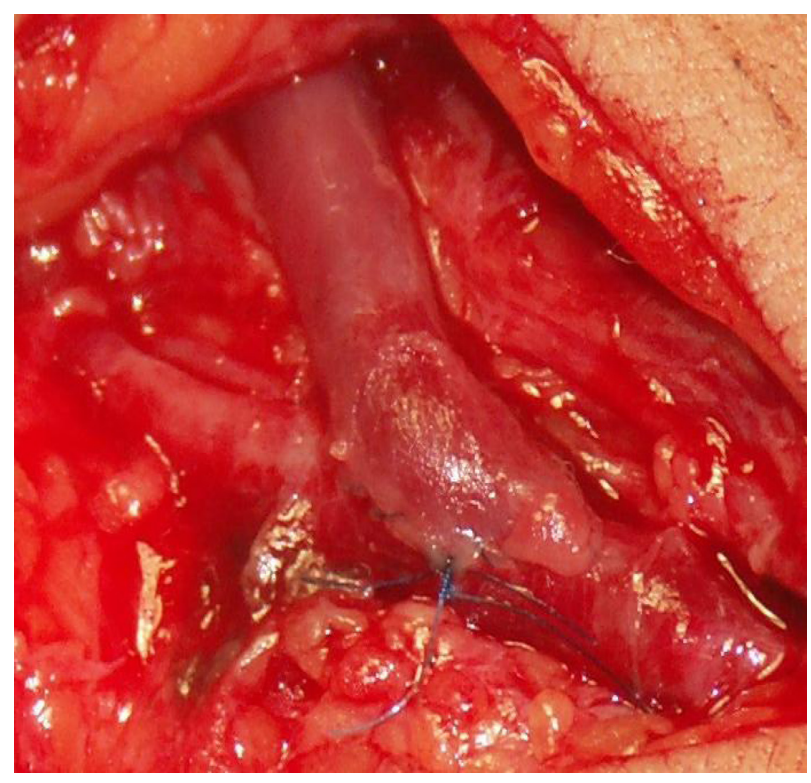

Figure I Native radio-cephalic arteriovenous fistula for hemodialysis, with lateroterminal anastomosis.

The patency rate for distal access at 1 year, reported in the literature, varies from $56 \%{ }^{7}$ to $79 \% .{ }^{8}$

The second treatment option is represented by the proximal AVF. It has the advantage of employing major caliber autologous material, which facilitates both the making up of the access and the subsequent venous cannulation for the use of access, as well as a higher patency rate compared with distal ones. However, it is characterized by a higher rate of complications such as steal syndrome and arterial alterations in cardiac output.

The brachio-basilic AVF also requires an additional technical procedure; that is, the superficialization of the basilic vein. ${ }^{9}$ This can be done in two stages, with the advantage of handling a vein which is already "arterialized" and therefore more resistant, but with the drawback of a more delayed use of the access.

One-year patency rate for proximal accesses reported in the literature varies from $70 \%{ }^{10}$ to $84 \% .{ }^{11}$

Before starting to use the AVF, a waiting time is needed in order to obtain structural modifications of the vein wall which consist of "arterialization" as a result of the turbulent flow. According to the guidelines NKF-K/DOQI $2006,{ }^{6}$ an access can be defined functional when the flow is $>600 \mathrm{~mL} / \mathrm{min}$, the vein has a minimum diameter of 0.6 $\mathrm{cm}$ and does not exceed the depth of $0.6 \mathrm{~cm}$, and the margins are clearly identifiable. The timing related to the achievement of these characteristics ranges from 1 to 3 months from surgical intervention of AVF. To evaluate the abovementioned parameters, a careful clinical and instrumental monitoring 
is required. In particular, the flow measurement method would be useful.

The most frequent complications related to AVFs are insufficient maturation of the AVF, stenosis, thrombosis, infection, aneurysm, "steal syndrome" due to ischemia, and high-rate flow AVF.

The failure of AVF can be related to stenosis of the artery of vein. Such a complication can be corrected by means of endovascular or surgical procedure, so short stenotic segments can be treated by means of percutaneous transluminal angioplasty, whilst surgical replacement is the gold standard for more extensive stenotic segments.

Nowadays, an increasing proportion of people who start dialysis are 75 years or older, with three-quarters of them having five or more comorbidities, and 90\% having cardiovascular disease. ${ }^{12}$ Indeed, when the radio-cephalic fistula was described in 1966 by Cimino and Brescia, the patients' average age was 43 years, almost all had chronic glomerulonephritis. A preoperative, clinical prediction ruled to determine fistulas that are likely to fail to mature showed the relevance of older age as a risk category for "fail to mature". ${ }^{13}$ For this reason, placement of unnecessary AVF in elderly patients with low life expectancy is not recommended.

However, in order to have tools to predict AVF maturation/failure or successful use, an ongoing multicenter clinical trial, "The Hemodialysis Fistula Maturation (HFM) Study", has been designed to elucidate clinical and biological factors associated with fistula maturation outcomes. ${ }^{14}$

\section{Arteriovenous graft (AVG)}

This type of VA consists of an AVF made with prosthetic interposition between an artery and a vein, with two purposes: the first is to be able to link two vessels which would not be possible to connect due to their distance, ${ }^{15}$ and the second is to interpose between an artery and a vein a high capacity prosthetic segment that can also be used for the insertion of HD catheters.

AVG is the second step of treatment, following the AVF made with native vessels. ${ }^{16}$ In selected cases, an AVG is indicated as the first line of treatment, such as in cases of paucity of autologous material and/or for a short predictable period of hemodialytic treatment (children), ${ }^{17}$ or in patients with short obese limbs, where the superficial veins are deep in the subcutaneous tissue, and finally in patients with extreme vascular fragility (thrombocytopenic purpura), where the simple venous puncture produces wounds and serious hematomas. ${ }^{18}$
The prosthetic AV access has been the most common access for dialysis in the US. This is related to several reasons and to a nihilistic attitude on the part of access surgeons that contributes to the underutilization of autogenous access sites. ${ }^{19-21}$ However, there are many efforts in the US and Canada to reverse this trend, as several studies suggest greater morbidity of AVG compared with autogenous access. $^{19-25}$

For optimal AVG planning, a clinical evaluation of the upper limb is necessary. Skin integrity, presence of superficial veins, which imply a central vein occlusion, and the presence of peripheral pulses should be evaluated. The second step for optimal planning is the duplex ultrasound exam. Vessel mapping is very important to reduce secondary surgical or endovascular procedures. ${ }^{26}$

Duplex ultrasound provides indications on upper limb artery patency and on the presence of stenosis or occlusions that could be treated before restoring an adequate flow. Outflow study is needed to evaluate vessel patency and diameter, which are predictive factors of failure.

Silva et $\mathrm{al}^{27}$ applied preoperative duplex ultrasound of both arterial inflow and venous outflow and concluded that a minimal vein diameter of $4 \mathrm{~mm}$ was required for a successful polytetrafluoroethylene-vein anastomosis.

\section{Graft materials}

Prosthetic AV grafts are classified as either biological or synthetic. In general, biological prostheses are of limited availability, expensive, and of variable size and quantity (Table 1). Benedetto et $\mathrm{al}^{28}$ described a technique to rescue surgery of autologous AVF using bovine mesenteric vein, with good results. These can be placed in the forearm, the arm, and the thigh, and can have a straight, curved, or loop configuration.

Although insertion sites in the upper limb are preferred because of the lower risk of associated sepsis, when the upper-limb sites are exhausted, the thigh is the next favored site. $^{29,30}$ Slater and Raftery ${ }^{31}$ reported a cumulative graft patency of $80.5 \%$ at 2 years, with no graft loss due to sepsis, in a series of 22 thigh grafts inserted in 21 patients. However, Englesbe et $\mathrm{al}^{32}$ reported a less favorable experience: $27 \%$ of the femoral AV grafts were lost for sepsis, with an overall secondary patency rate of $26 \%$ at 2 years. When implanted in the thigh, the graft can have a straight, looped, or curved configuration.

Forearm grafts with loop configuration yield greater overall patency rates and require fewer revisions than forearm grafts with straight configuration. ${ }^{33}$ Axillary loop 
Table I Graft materials

\begin{tabular}{|c|c|c|}
\hline Type & Material & Characteristic \\
\hline \multirow[t]{5}{*}{ Biological } & Denatured homologous vein allograft & \\
\hline & Cryopreserved saphenous vein & Caution should be exercised in patients at high risk for infection. \\
\hline & Bovine heterografts - typified by SGVG 100 & $\begin{array}{l}\text { Safe alternative for patients with a history of multiple failed } \\
\text { synthetic grafts. }{ }^{25}\end{array}$ \\
\hline & Human umbilical vein & \\
\hline & Sheep collagen grafts & \\
\hline \multirow[t]{5}{*}{ Synthetic } & Dacron $^{\circledR}$ (E.I. du Pont de Nemours and Company, & \\
\hline & Wilmington, DE, USA) & \\
\hline & PTFE & $\begin{array}{l}\text { This fluorocarbon polymer has become the prosthetic graft of } \\
\text { choice. Stretch ePTFE is preferable to standard ePTFE. }\end{array}$ \\
\hline & Procol $^{\circledR}$ (Hancock, Jaffe, Laboratories, Irvine, CA, USA) & Higher graft survival for the bioprosthesis versus ePTFE \\
\hline & $\begin{array}{l}\text { bovine mesenteric vein graft, which closely resembles the } \\
\text { human saphenous vein }\end{array}$ & $(82 \%$ versus $50 \% ; P<0.04)$ over a period of 20 months. \\
\hline
\end{tabular}

Abbreviations: ePTFE, expanded PTFE; PTFE, polytetrafluoroethylene; SGVG 100, SynerGraft Vascular Graft Model I00.

grafts are indicated when more distal options for VA are exhausted or when the risk of steal syndrome is extremely high (Figure 2).

\section{AVG complications}

Functional survival of AVG is much shorter than with AVF. The natural course of AVG is thrombosis due to venous stenosis caused by neointimal hyperplasia. The increased production of smooth muscle cells, myofibroblasts, and vascularization within the neointima is the main cause of thrombosis. There is also angiogenesis and numerous macrophages in the tissue around the graft. ${ }^{34}$

Growth factors such as platelet derived, vascular endothelial, and basic fibroblast growth factors are present within the neointimal lesion. Thrombosis of an AVG is usually the result of multiple factors, such as stenosis, hypotension, and excessive compression for hemostasis. The risk for thrombosis increases with decreasing blood

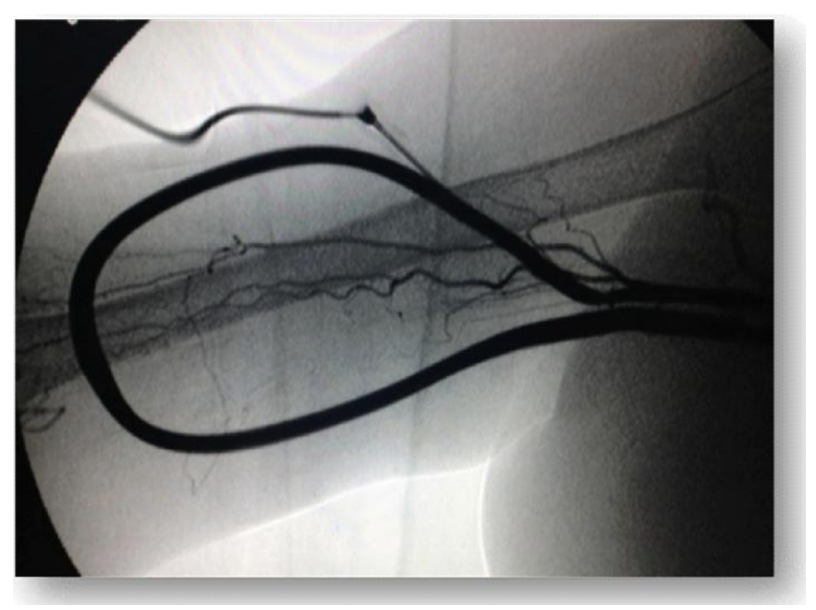

Figure 2 Synthetic axillo-axillary graft in polytetrafluoroethylene material. flow. ${ }^{35}$ The influence of the anastomotic angle upon hemodynamics has been investigated using a porcine aortic model with $8 \mathrm{~mm}$ polyurethane interposition grafts and an end-to-side configuration. Distal anastomoses were created, with angles of either $90^{\circ}, 45^{\circ}$, or $15^{\circ}$. Both the $90^{\circ}$ and $45^{\circ}$ configurations displayed a zone of recirculation at the anastomosis, while the $15^{\circ}$ anastomosis displayed no flow disturbance. ${ }^{36}$

To reduce the risk of graft thrombosis, the use of dypiridamole, sulfinpyrazone, ticlopidina, and combined aspirin and dipyridamole has been proposed. ${ }^{37}$ Although these agents showed a low rate of serious bleeding in dialysis patients, there is no definitive evidence of their efficacy. ${ }^{37}$ The effect of fish oil on synthetic HD graft patency was studied in a recent clinical trial. The authors showed that daily fish oil ingestion failed to reach the primary outcome since it did not decrease the proportion of grafts with loss of native patency within 12 months. However, other secondary outcomes such as graft patency, rates of thrombosis, and interventions, were improved. ${ }^{38}$

AVP infections are serious complications and are the second leading cause of dialysis access loss. The incidence of HD-related bacteremia is more than tenfold higher in AVGs than AVFs: 2.5 episodes per 1,000 dialysis procedures versus $0.2 .{ }^{39}$ Patients must be more careful about their hygiene because it seems to be the most important modifiable risk factor. $^{40}$

The critical issues in the management of AV graft infection are the need to eradicate infection and to achieve HD with reduced morbidity. Treatment involves intravenous antibiotics and total graft excision in septic patients or when the graft is bathed in pus; subtotal, when all of the graft is removed except an oversewn small cuff of prosthetic 
material on an underlying patent vessel; and partial, when a limited portion of AVG is removed and a new graft is inserted through adjacent sterile tissue. ${ }^{41}$

Pseudoaneurysms should be referred to a surgeon for resection when they are $>2$ times wider than the graft or rapidly increasing in size or when the overlying skin appears under duress. ${ }^{42}$

Ischemia as a result of access placement is more common for AVGs than AVFs: vascular steal syndrome and ischemic monomelic neuropathy are two important clinical entities to be distinguished. Endovascular treatment with stent grafts in complicated access, in AVFs as well as in AVGs, is a simple, safe, and rapid ambulatory procedure that enables treatment of both the aneurysm and its accompanying draining vein stenosis. It enables continued cannulation of the existing access and avoids the use of central catheters. ${ }^{43,44}$

\section{Central venous catheters (CVCs)}

CVC represents a good choice, especially when urgent or emergent HD is required either at the time of initiation of renal replacement therapy or when a permanent access becomes dysfunctional. ${ }^{45}$ These devices are universally available, can be inserted into different sites of the body, and maturation time is not required, allowing immediate HD.

Preferable locations for insertion are the internal jugular and femoral veins, and in the third instance, the subclavian vein (Table 2). Ultrasonography accurately locates the target vein and also provides information about venous pressure and the presence of intravascular thrombi. Its use should therefore be an integral part of central venous catheterization. ${ }^{46}$

\section{Internal jugular vein (IJV)}

The IJV represents the first choice for CVC insertion for several reasons (Table 2). First of all, it is a large superficial vein that has easy ultrasound visualization. Moreover, the straight course into the superior vena cava or right atrium, without any corners, reduces the requirement for screening during insertion and allows high blood flow for HD. The lower part of the IJV lies behind a triangle formed by the junction of the sternal and clavicular insertions of the sternomastoid muscle and the clavicle. This triangle is used as a surface landmark (Figure 3).

Normally, the vein lays anterolaterally to the artery, but in a small percentage of patients, the vein is immediately anterior to the artery or even medial to it (Figure 4). ${ }^{47}$ Therefore, for the significant anatomical variation in the vein and its course, percutaneous ultrasound-guided technique for IJV access has become the standard practice. ${ }^{48}$

Traditionally, the vein has been located by the landmark technique; however, ultrasound guidance is now recommended as the preferred method for insertion of CVCs into the IJV in adults and children in elective situations (Figure 5). ${ }^{49}$ For the insertion of a CVC into the IJV, the patient should be optimally positioned, with a $10^{\circ}$ headdown tilt (Trendelenburg position) to help distend the vein and reduce the risk of air embolism. ${ }^{50}$

It may be safer if the patient's head is in the neutral position. Furthermore, the vein can lie directly above the carotid artery, increasing the risk of arterial puncture. A very modest degree of rotation of the head away from the side to be cannulated may be necessary, but extreme rotation is best avoided as it may reduce vein diameter. ${ }^{51}$ Indeed, head rotation can cause the IJV to move laterally in relation to surface landmarks and become more difficult to locate. ${ }^{52}$

There are two different main approaches, according to the visualization of the needle during its entry into the vein, using ultrasound guidance: in-plane and out-of-plane, placing the probe on the vein long axis or short axis. Recently, it has been shown that the lateral short axis in-plane technique has virtually no limitations, ensuring most benefits, and for this

Table 2 Central vein approaches for dialysis catheters

\begin{tabular}{|c|c|c|c|}
\hline Priority & Vein & Advantages & Disadvantages or complications \\
\hline \multirow[t]{3}{*}{ First choice } & Internal jugular vein & Best with ultrasound & Medium infection risk \\
\hline & & Right side gives more chance to & Medium bleeding risk \\
\hline & & correct blind catheter tip placement & Uncomfortable when not tunneled \\
\hline \multirow[t]{3}{*}{ Second choice } & Femoral vein & Lower bleeding risk & Higher infection risk \\
\hline & & No need for radiological control & Higher thrombosis risk \\
\hline & & after insertion & $\begin{array}{l}\text { Poor catheter performance when patient } \\
\text { sits up }\end{array}$ \\
\hline \multirow{5}{*}{$\begin{array}{l}\text { Third choice (avoid proximal } \\
\text { or terminal arteriovenous } \\
\text { fistula in the same side) }\end{array}$} & Subclavian vein & Lower infection risk & Higher bleeding risk \\
\hline & & Suitability for subcutaneous & Higher pneumothorax risk \\
\hline & & tunneling and port access & Higher thrombosis risk \\
\hline & & & "Blind" procedure that cannot be guided \\
\hline & & & with ultrasound \\
\hline
\end{tabular}




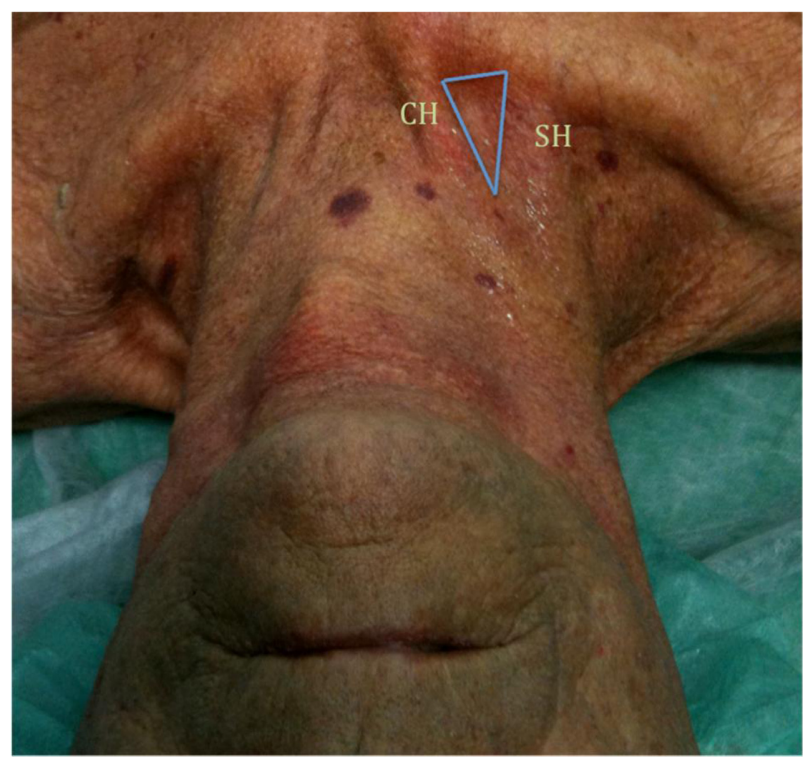

Figure 3 Photograph of neck in a malnourished patient demonstrating surface anatomy.

Note: It shows Sedillot's triangle, formed by the sternal $(\mathrm{SH})$ and clavicular $(\mathrm{CH})$ heads of the sternocleidomastoid. Inside this triangle is the approximate normal course of the internal jugular vein.

reason, it should be considered as the first-line technique for IJV cannulation. ${ }^{53}$

The common method for direct insertion into the great veins is the Seldinger technique, using a guidewire over the needle. With this technique after the vein is entered, the guidewire is advanced into the vein and the needle is removed. Once the guidewire has been passed, it is important not to insert too far. Indeed, it may irritate the right atrium and cause arrhythmias, most commonly atrial ectopics. Then, before inserting the catheter, a dilator needs to be passed over the guidewire, using care to not cause vein trauma. ${ }^{54}$

The length of the catheter inserted via the right IJV is typically $15 \mathrm{~cm}$, whilst it should be $17 \mathrm{~cm}$ via the left IJV. Ideally, for temporary catheters, the tip should lie outside the right atrium, and its position should be checked during the procedure with electrocardiography, or on a post-procedure chest radiograph, before starting HD. For tunneled cuffed catheter, one of the two tips should lie inside the right atrium,

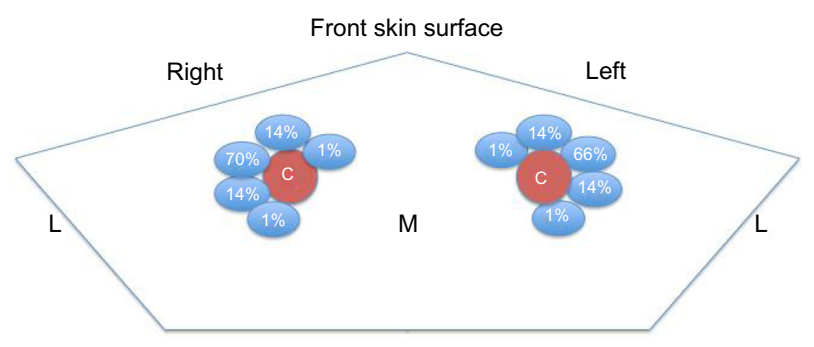

Figure 4 Percentage of variation in anatomical relations between the right and left internal jugular vein (in blue) and common carotid artery (C).

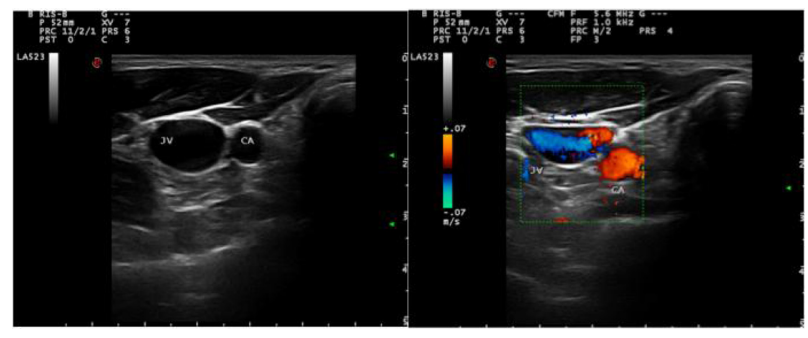

Figure 5 Ultrasound cross-sectional (left) and Doppler ultrasound (right) image of right internal jugular vein (IJV) and carotid artery (CA).

Note: Both vessels are very superficial since they are in a range of depth of field between I and $2.5 \mathrm{~cm}$.

whilst the other tip should lie $1 \mathrm{~cm}$ above, outside the right atrium.

\section{Femoral vein}

The femoral vein is considered the second approach for inserting temporary dialysis catheters in inpatients. The advantage is lower bleeding risk, and moreover, radiological control after insertion is not required (Table 2). However, $\mathrm{X}$-ray verification may be useful for longer-term access to ensure that there is no kinking and that the catheter tip has not entered lumbar vein or other branches. The landmark technique was described for the first time by Hohn and Lambert in 1966. The patient is in a supine position and abducts and externally rotates the thigh. The point of needle insertion into the femoral vein is situated below the inguinal ligament (approximately $2 \mathrm{~cm}$ ) and medially to the beating of the femoral artery. The needle is inserted cephalad at an angle of $10^{\circ}-15^{\circ}$ dorsally in relation to the frontal plane and slightly medially in relation to the sagittal plane, and it is usually entered at about $2-4 \mathrm{~cm}$ deep. The abovementioned Seldinger technique is used also for the femoral vein.

The Valsalva maneuver is used to increase femoral vein diameter. The optimal location of the distal tip of catheters inserted through the iliac veins should be the inferior vena cava or the right atrium. However, the majority of standard catheters $(20 \mathrm{~cm}$ long) reach the iliac veins, and this position of the tip can cause increased blood recirculation. It should be taken into consideration that longer catheters increase the resistance of blood flow. For the permanent CVCs in femoral veins, a possible alternative is the external abdomen location of a cuffed catheter, as a variant of the normal external leg location (Figure 6).

\section{Subclavian vein}

The subclavian insertion of the catheter is considered the third choice because of the high risk of subclavian thrombosis with complication to create a VA in the ipsilateral arm (Table 2). 


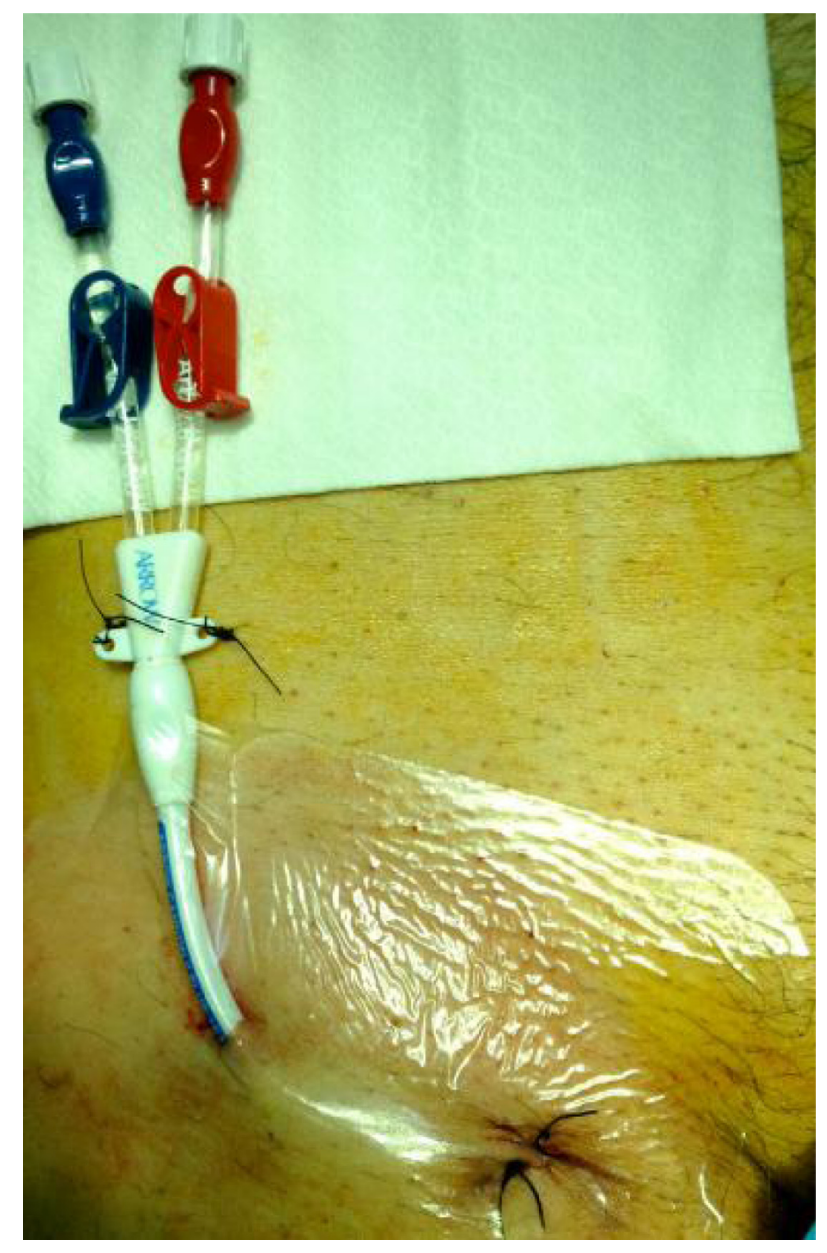

Figure 6 External abdomen location of cuffed tunneled central venous catheters in femoral vein, as a variant of the normal external leg location.

Historically, the supraclavicular subclavian catheterization was realized by Yoffa. ${ }^{55}$ The objective of this technique is to puncture the subclavian vein in its superior aspect just as it joins the IJV. The correct identification of the clavisternomastoid angle formed by the junction of the lateral head of the sternocleidomastoid muscle and the clavicle is mandatory. Active raising of the patient's head may make this landmark more apparent. The needle is inserted $1 \mathrm{~cm}$ lateral to the lateral head of the sternocleidomastoid muscle and $1 \mathrm{~cm}$ posterior to the clavicle and directed at a $45^{\circ}$ angle to the sagittal and transverse planes and $15^{\circ}$ below the coronal plane, aiming toward the contralateral nipple. ${ }^{56}$ The needle bisects the clavisternomastoid angle as it is advanced in an avascular plane, away from the subclavian artery and the dome of the pleura, entering the junction of the subclavian vein and IJV. The right side is preferred because of the lower pleural dome, more direct route to the superior vena cava, and absence of thoracic duct. The Trendelenburg position is recommended to decrease risk of air embolism and to potentially help to distend the vein, as the subclavian vein is not bound by fascia on its superior aspect. To further minimize complications, the needle bevel should be facing down prior to insertion, attempts should cease after 2-3 unsuccessful tries, and most importantly, the clavisternomastoid angle must be clearly identified prior to insertion. The main disadvantages are higher bleeding and pneumothorax and thrombosis risk. Moreover, a "blind" procedure cannot be guided with ultrasound (Table 2).

\section{CVC complications}

Caution needs to be used in implanting and management of CVCs, since their use is associated with a high risk of complications. ${ }^{56}$ Complications associated with CVC insertion range from 5\% to $19 \% .{ }^{57,58}$ Insertion complications include vascular injury (arterial puncture, pseudoaneurysm, and AVF), hematoma, air embolism, pneumothorax, and malposition. Generally, all these complications are limited to accidental arterial puncture when ultrasound guidance is used. ${ }^{48,59}$

Arterial puncture is a common risk during vein cannulation, since veins run alongside arteries. Even if the risk is higher for femoral than for jugular and subclavian veins, the complications of subclavian arterial puncture are much more severe, as the vessels cannot be compressed manually from the outside of the body because they lie under the clavicle, and this leads to hemothorax in severe cases.

The risk of pneumothorax is greatest in the subclavian area due to the proximity of the pleura to the vein, with an incidence rate of $2 \%-3 \%$ with this approach. ${ }^{57}$

Indwelling complications are infection, thrombosis, catheter pinching/kinking, and fracture with possible embolization. Infections are discussed elsewhere. The risk of thrombosis is lower in the IJV, slightly higher in the subclavian vein, and still higher in the femoral vein. ${ }^{60}$ Classically, thrombosis is more likely where there is the combination of low blood flow, turbulence, and increased coagulopathy. The severity of thrombosis depends on the sites of location. Indeed, thrombosis of superficial veins in the forearm causes mild morbidity, whereas femoral venous thrombosis may cause life-threatening pulmonary embolism.

Another complication is stenosis of veins that may occur over a period of time, after damage to the vein wall due to infection or mechanical stress. The risk of stenosis is reduced if the catheter lies in the center of a big vein with a high blood flow away from junctions with other veins.

Puncture of the carotid artery during attempted IJV cannulation can cause emboli of atherosclerotic tissue into 
the brain, with the severe consequences of a stroke. Arterial emboli from the subclavian and femoral regions are less dangerous to the patient.

Infections are more common in the femoral region due to the proximity of the perineum, whilst the subclavian vein probably causes less infection than the IJV. ${ }^{57}$

Since an increasing number of patients have implanted pacemakers and defibrillators, usually inserted via the subclavian veins and superior vena cava into the right heart, a careful assessment of risks and benefits should be taken. The access site should be on the opposite side to where the implanted device lies wherever possible. However, there is a risk of superior vena cava syndrome due to thrombosis of the vessel secondary to placement of CVCs or pacemakers. ${ }^{61}$

\section{Temporary and permanent dialysis catheter}

CVCs for HD are essentially of two types: acute (non-tunneled) catheters and chronic (tunneled) catheters. The choice between placement of an acute/temporary or a chronic/permanent catheter should be based on several factors: duration of use, bacteremia, and patient conditions.

Acute dialysis catheters are non-cuffed, non-tunneled catheters used for immediate VA. They are primarily used for acute renal failure in bed-bound patients, and for short-term use in patients with malfunction of permanent access. Long-term use of acute catheters is not recommended, but does occur, with acceptable infection rates, in dialysis centers where tunneled, cuffed catheters are not available. Most acute catheters are made of polyurethane, available with larger lumen sizes and capable of delivering blood flow rates over $300 \mathrm{~mL} / \mathrm{min}$ following NKF-K/ DOQI guidelines.

Concerning the indwelling time for catheter access, the acute catheter lacks a subcutaneous cuff, and it should be restricted to the first 1 or 2 weeks of HD, knowing that beyond 1 week, the infection rate increases exponentially. Moreover, guidelines recommend that temporary catheters should remain in place no longer than 5 days at the femoral vein. ${ }^{6}$

A chronic catheter has a subcutaneous cuff which is placed in the subcutaneous tissue near the insertion site of a tunneled catheter and allows for fibrous sealing of its skin entry; this provides a barrier against infection by preventing migration of bacteria down the outer surface of the catheter, and the catheter can potentially be used for months to years. Insertion of a cuffed, tunneled catheter is recommended as soon as it is known that prolonged renal replacement therapy (more than 2 weeks of HD) is needed.

\section{Catheter materials}

Materials play an important role in terms of indwelling time of the catheter. During the past decade, there has been an emergence of technological advancements in the design of dialysis catheters in an attempt to reduce catheter malfunction, decrease infection rates, and improve their longterm efficiency. The availability of plastic polymers such as polyethylene, polypropylene, polyvinyl chloride, and fluorocarbons (polytetrafluoroethylene) provided tubing that began to meet many of the properties required for intravascular implantation. These materials are relatively thrombogenic by present day standards and also quite rigid, contributing to endothelial injury. ${ }^{62}$

Polyvinyl chloride may be rendered more flexible by adding plasticizers, but these compounds elute into blood, with the possibility of unwanted biological effects and progressive hardening of the catheter. In the early 1940s the development of silicone polymers provided materials that offered greater biocompatibility and stability for long-term implantation, particularly due to reduced thrombogenicity. ${ }^{63}$

By the early 1960s, medical grade silicone tubing had become commercially available - a significant advance in the evolution of clinical and experimental vascular catheters. ${ }^{64}$ More recently, developments in biocompatible polyurethane materials have provided catheter materials with physical properties superior to silicones. ${ }^{65}$

Today, the most important materials used for CVCs are silicon and polyurethane, both of which are biocompatible and durable. There is no significant difference in the overall duration of function between silicone and polyurethane catheters; however, it has been observed that the infection rates were 3.6 per 1,000 catheter-days for silicone catheters and 3.5 per 1,000 catheter-days for polyurethane catheters. ${ }^{66}$

The main difference between these materials is that polyurethane has a higher tensile strength than silicone, which allows catheters to be manufactured with a higher inner lumen and same outer diameter, improving in that way the overall catheter flow rate. Perhaps, due to the thinner walls, polyurethane catheters are more prone to kinking, although industry has already overcome this problem, offering kinkresistant double-lumen polyurethane catheters with flows greater than $400 \mathrm{~mL} / \mathrm{min}^{67}$

One strategy aimed at reducing infection rates in acute catheters was the addition of an antimicrobial coating effective against pathogens. A study by Rupp et $\mathrm{al}^{68}$ demonstrated a protective effect in the prevention of bacterial colonization when comparing protected with unprotected catheters. 
Protected catheters were able to reduce bacterial colonization of the catheter by $44 \%$ and catheter-related bacteremia by $79 \%{ }^{68,69}$

Currently, many new dialysis catheters are being developed in an effort to decrease thrombosis and infection rates and to prolong the long-term outcome of catheterization. Thrombosis has long been a problem with dialysis catheters. One way this problem has been addressed is by the evolution of material technology. A transition has been made using polyurethane or Carbothane $\mathrm{TM}^{\mathrm{TM}}$ (a polyurethane/polycarbonate copolymer; the Lubrizol Corporation, Wickliffe, OH, USA) rather than silicone because it allows for better catheter resistance and softness, while still maintaining a large internal diameter.

Recirculation is another important issue with chronic HD catheters. Correct tip positioning and design are two key points to reduce or prevent recirculation.

In our experience, retrograde tunneling improves the ability to ideally position the catheter tip, cuff and hub, and split-tip design, with both lumens placed in the right atrium. Retrograde tunneling has always been a good option to provide high blood flow with less recirculation, overcoming limits of some step-tip catheters, mainly due to the distance between arterial and venous port.

Recently, the latest technology has been able to provide a unique tip design, featuring ports that are reversed with respect to conventional step-tip (or staggered-tip) catheters. In fact, the arterial intake port, which is located at the distal tip of the catheter, is positioned in the lower right atrium and the venous outflow port is $6 \mathrm{~cm}$ proximal to the arterial port. The positioning of the arterial port directly above the inferior vena cava, in combination with the port spacing, minimizes recirculation, maintaining the advantages of the correct tip positioning of a retrograde catheter.

\section{Vascular access (VA) infections}

Patients with end-stage renal disease requiring dialysis with VA through CVC are at increased risk of infection. Infection is responsible for the removal of about $30 \%-60 \%$ of HD CVCs, and hospitalization rates are higher in $\mathrm{CVC}$ patients than $\mathrm{AVF}$ patients. ${ }^{70}$ Furthermore, CVC dialysis patients face a risk of death from infection, $41 \%$ higher than those using AVF. ${ }^{71}$

Catheter-related infections can be localized or systemic. In the first case, the infection may affect the CVC insertion site or may spread to the subcutaneous route. Exit-site infection has the highest incidence in hemodialyzed patients, especially in short-term CVC patients. It is characterized by erythema, tenderness, induration, or exudate within $2 \mathrm{~cm}$ from the exit site. In tunnel infection erythema, tenderness, induration, or exudate are present at more than $2 \mathrm{~cm}$ distance from the exit site or along the subcutaneous route of the tunneled CVC. ${ }^{72}$

The most dangerous infectious complication is catheterrelated bloodstream infection (CRBSI), associated with high rates of morbidity and mortality, and adding excessive costs to the care of these patients. ${ }^{73}$ A systematic review highlighted how patients using CVC for HD face a higher risk of CRBSI compared with patients who use it for other reasons. ${ }^{74}$

In the United States in 2007-2008, the rate of pooled men access-related bloodstream infection in HD patients with a central line was 1.05 cases per 1,000 catheter days. ${ }^{75}$ In order to cause infections, microorganisms have to access the extra-luminal or intra-luminal surface of the catheter, where they merge with a biofilm. Microorganisms reach the CVC through the percutaneous route at the time of insertion or a few days afterwards, or they can contaminate the catheter hub (and lumen) when the catheter is inserted over a percutaneous guidewire or when it is later manipulated. The first instance is most frequently the cause of short-term CVC infections, whereas the second is responsible for intraluminal colonization of long-term catheters. Less frequently, organisms are carried hematogenously to the implanted CVC from remote sources of local infection or contaminated fluids. ${ }^{76}$

The pathogens which are mainly responsible for infections are Staphylococcus, Gram-negative enteric bacilli, Pseudomonas aeruginosa, and Candida spp. ${ }^{77}$ These pathogens are similar in that they can form a biofilm on the CVC walls, which makes them very resistant to antibiotic action.

Exit-site infections without fever may be treated with local antibiotic application, and if the patient does not recover from infection, they will be treated with systemic antibiotics. If the antibiotic fails, the catheter should be removed. Tunnel infections demand CVC removal and systemic antibiotic treatment. Systemic infections such as bloodstream infection are definitely more critical, and they are also harder both to diagnose and to treat.

The chance that a patient with CVC may have developed a CRBSI must be taken into account whenever there is fever, shivers, or hypotension and, furthermore, any other possible causes of infection are lacking. Several diagnostic methods allow us to diagnose CRBSI. Most of them use quantitative or semi-quantitative cultures of CVC segments or blood cultures taken simultaneously from the CVC and from a peripheral vein. ${ }^{78}$

A simple method that can be carried out in most laboratories is based on the assessment of the positivization 
time difference of cultures with the same blood quantity taken simultaneously from CVC and peripheral vein: the positivization of a blood culture taken from CVC, at least two hours earlier than that taken from a peripheral vein indicates CVC related sepsis. ${ }^{79}$

In patients undergoing $\mathrm{HD}$ who have a tunneled $\mathrm{CVC}$, the CRBSI diagnosis is more complicated, since carrying out peripheral blood cultures is made harder because of both the lack of accessible peripheral veins and the need to avoid puncturing veins that in the future could be used for the creation of an AVF. Other complications are linked to frequent fever onset during dialysis. In this case, there may not be a remarkable difference in bacterial concentration between the blood taken from a peripheral vein and that taken from a CVC (or dialysis circuit) because all blood goes through the CVC. Moreover, in an outpatient setting, there may be delays in blood sample incubation, and in addition, it would prove even more difficult to exclude any other possible causes of infection. ${ }^{80}$ CRBSI in HD patients presents a series of characteristics that may result in a different treatment compared with that of other patients.

The guidelines of Infectious Disease Society of America, ${ }^{72}$ of the National Institute for Health and Clinical Excellence, ${ }^{81}$ and the position statement of European Renal Best Practice $^{82}$ provide detailed advice about CRBSI prophylaxis and management.

In the case of CRBSI, it is necessary to medicate promptly with antibiotics and to take into consideration CVC removal. The required therapy depends on several factors, among which a major role is played by the patient's clinical conditions, the kind of catheter (short or tunneled), and the availability of a new site for the insertion of a new catheter, and last but not least the pathogen responsible for the infection.

In the case of patients with a non-tunneled CVC having fever and mild-to-moderate diseases (no hypotension or organ failure), it is not strictly necessary to remove the CVC. It is essential to carry out blood cultures both from CVC and peripheral vein and to consider an antibiotic therapy that will be necessary in case of positive blood cultures.

In the case of seriously ill patients (hypotension, hypoperfusion, or signs and symptoms of organ failure) with a non-tunneled CVC, blood cultures from the CVC and peripheral vein must be carried out, and the CVC must be removed and inserted in a new site or exchanged over a guidewire; antibiotic therapy must be initiated promptly. The tip of the removed CVC must be sent for culture, and in the case of a positive result, the new CVC should be replaced again.
CRBSI patients with a tunneled HD catheter may be managed in different ways. It is possible to keep the CVC and start an antibiotic therapy. However, this strategy is saddled with the frequent recurrence of bloodstream infection at the end of the therapy and by a high probability of failure.

Better therapy results are obtained with the removal and the substitution of the CVC. Catheters should be exchanged as soon as possible and within 72 hours of initiating antibiotic therapy in most instances, and such exchange does not require a negative blood culture result. ${ }^{6}$

Even though the catheter substitution is clinically advised, prior to the removal of a catheter in HD patients it is necessary to make sure that a new site is available for the insertion of a new catheter. A strategy to maintain the catheter in situ is to join the systemic therapy with the lock therapy for 3 weeks.

Antibiotic lock therapy consists of the instillation of a highly concentrated antibiotic solution into an intravascular catheter lumen for the purpose of sterilization in order to treat CRBSI, minimize associated complications, and avoid catheter removal. ${ }^{83}$ An evaluation of the efficacy of such therapy must be carried out 3 days later. If, after this period, fever, bacteremia, or fungemia still persist, it is necessary to remove the CVC and continue the systemic treatment. If there is no fever and the cultures are negative, the systemic therapy will be continued and joined to the lock therapy or, alternatively, the CVC will be substituted on a guidewire.

The empiric antimicrobial therapy must be wide spectrum and active against Gram-positive (especially staphylococci) and Gram-negative bacilli. Empiric antibiotic coverage for both Gram-positive and Gram-negative bacteria should be provided. If the patient shows different risk factors for candidemia (such as previous use of antibiotics or steroids, previous abdominal surgery, or parenteral nutrition), the use of antifungal medication is required. A more specific therapy will be started as soon as the data about the isolation and sensitivity of the responsible pathogen is available.

The ideal antibiotic for the treatment of HD CRBSI must 1) be active towards those pathogens that are usually responsible for infection; 2) have a fast bactericide action; 3) have concentration-dependent action; 4) not be cleared via the kidneys; 5) have a long biological half-life that allows a single, daily administration after HD; and 6) have a good capacity of penetration into the biofilm.

Cefazolin is particularly effective in the case of infection from methicillin-sensitive staphylococci, whereas daptomycin has features that make it particularly indicated in the case of methicillin-resistant staphylococci. Against 
Gram-negative, the best antibiotics are ceftazidime, aminoglycosides, and carbapenems, whereas echinocandins and liposomal amphotericin B will be used in CRBSI deriving from fungi.

The therapy duration varies in relation to the isolated pathogen and to the removal or keeping of the CVC. Short therapies (5-7 days) are sufficient for the treatment of CRBSI provoked by coagulase negative staphylococci if the catheter is removed, while longer treatments (up to 4-6 weeks) are necessary for CRBSI with complications (suppurative thrombophlebitis or other metastatic infections such as lung or brain abscesses, osteomyelitis, and endocarditis) from Staphylococcus aureus.

Several interventions have proved to be effective in CRBSI prevention. The core interventions for bloodstream infection prevention in dialysis facilities are indicated by Atlanta Centers for Disease Control and Prevention in nine recommendations regarding continue efforts to reduce the use of a catheter for HD to a minimum: periodic surveillance for bloodstream infection, hand hygiene observation, catheter/ vascular access care observation to assess staff adherence to aseptic technique when connecting and disconnecting catheters and during dressing changes, staff education and competency, patient education/engagement, catheter reduction, chlorhexidine for skin antisepsis, catheter hub disinfection, the application of antibiotic ointment or povidone-iodine ointment to catheter exit-site change. ${ }^{84}$

\section{Conclusion}

A well-functioning VA remains the Achilles' heel of HD and is essential, since a good VA translates into an efficient HD procedure. Expenditures for access care constitute a large fraction of the total cost of caring for HD patients. The creation of an acceptable VA does not always result in permanent access availability because of numerous complications. Difficulties in maintaining VA are the main challenge for nephrologists and nurses operating in dialysis units. Proper VA maintenance involves good cooperation between medical care personnel and patients. A full understanding of the etiology of access failure requires an evaluation of numerous factors, including patient demographics, fistula type, and patient compliance with fistula care. An important cause of VA failure is acute and chronic thrombosis. For this reason, to prevent thrombotic events of VA, it is important to monitor biomarkers of coagulation activation. Several biomarkers have been studied, such as thrombin-antithrombin (TAT), D-dimer, von Willebrand factor, PAI-1 (plasminogen activator inhibitor-1 antigen), and soluble p-selectin. ${ }^{85} \mathrm{~A}$ recent comparative study performed in $70 \mathrm{HD}$ patients showed that TAT, D-dimer, von Willebrand factor, p-selectin, and hsCRP (high-sensitivity C-reactive protein) were all elevated in patients on HD compared with controls. However, only TAT levels increased and inversely correlated with primary assisted patency and secondary patency. ${ }^{86}$

Despite emerging vascular graft technologies and permanent cuffed catheters, the basic autogenous AV fistula described by Brescia and Cimino remains the first choice for chronic HD. It is the best access for longevity and lowest association with morbidity and mortality. For this reason, guidelines from different countries strongly recommend AVF use. .2, $87,88^{-10}$

Once autogenous options have been exhausted, prosthetic fistulae become the second option of maintenance HD access alternatives. CVCs have become an important adjunct in maintaining patients on HD. However, their use is linked to higher rates of infection and could compromise dialysis adequacy.

The main factors leading to high use of catheter as chronic access in some countries suggest that VA patient preference may be influenced by sociocultural factors. Indeed, catheter preference was greatest among current and former catheter users, suggesting that one way to reduce barriers to successful use of AVF may be to avoid catheter use whenever possible. $^{2}$

Moreover, the fistula-first/catheter-last approach to the optimal access type for HD was recently revised due to the existence of selection bias in studies comparing clinical outcomes by VA type. ${ }^{89}$ In particular, it was observed that healthier patients are more likely to use an AVF for $\mathrm{HD}$, whilst patients who need urgent dialysis and who are ineligible for fistula are more likely to use a CVC. Therefore, the true risk attributable to access type may be masked by this selection bias. However, some author observed that after adjustment for health status, the advantage of AVF still persists. ${ }^{90}$

In conclusion, proper VA maintenance requires integration of different professionals to create a vascular access team. Such a team should include a nephrologist, radiologist, vascular surgeon, infectious disease consultant, hematologist, and members of the dialysis staff. They should provide their experience in order to give the best options to uremic patients and the best care for their VA.

\section{Disclosure}

The authors declare no conflicts of interest in this work. 


\section{References}

1. Vascular Access 2006 Work Group. Clinical practice guidelines for vascular access. Am J Kidney Dis. 2006;48 Suppl 1:S176-S247.

2. Fissell RB, Fuller DS, Morgenstern H, et al. Hemodialysis patient preference for type of vascular access: variation and predictors across countries in the DOPPS. J Vasc Access. 2013;14(3):264-272.

3. Malovrh M. Native arteriovenous fistula: preoperative evaluation. Am J Kidney Dis. 2002;39(6):1218-1225.

4. Santoro D, Savica V, Bellinghieri G. Vascular access for hemodialysis and cardiovascular complications. Minerva Urol Nefrol. 2010;62(1):81-85.

5. Mendes RR, Farber MA, Marston WA, Dinwiddie LC, Keagy BA, Burnham SJ. Prediction of wrist arteriovenous fistula maturation with preoperative vein mapping with ultrasonography. J Vasc Surg. 2002;36(3):460-463.

6. National Kidney Foundation, Inc. K/DOQI Guidelines - Updates 2006. New York: National Kidney Foundation, Inc; 2001. Available from: http://www.kidney.org/PROFESSIONALS/kdoqi/guideline_upHD_ PD_VA/index.htm. Accessed June 5, 2014.

7. Leapman SB, Boyle M, Pescovitz MD, Milgrom ML, Jindal RM, Filo RS. The arteriovenous fistula for hemodialysis access: gold standard or archaic relic? Am Surg. 1996;62(8):652-656.

8. Wolowczyk L, Williams AJ, Donovan KL, Gibbons CP. The snuffbox arteriovenous fistula for vascular access. Eur J Vasc Endovasc Surg. 2000;19(1):70-76.

9. Korkut AK, Kosem M. Superficialization of the basilic vein technique in brachiobasilic arteriovenous fistula: surgical experience of 350 cases during 4 years period. Ann Vasc Surg. 2010;24(6):762-767.

10. Livingston CK, Potts JR 3rd. Upper arm arteriovenous fistulas as a reliable access alternative for patients requiring chronic hemodialysis. Am Surg. 1999;65(11):1038-1042.

11. Bender MH, Bruyninckx CM, Gerlag PG. The brachiocephalic elbow fistula: a useful alternative angioaccess for permanent hemodialysis. J Vasc Surg. 1994;20(5):808-813.

12. US Renal Data System: USRDS 2005 Annual Data Report: Atlas of Endstage Renal Disease in the United States. Incidence and Prevalence; Patient Characteristics, Bethesda, National Institutes of Health, National Institute of Diabetes and Digestive and Kidney Diseases, 2005.

13. Lok CE, Allon M, Moist L, Oliver MJ, Shah H, Zimmerman D. Risk equation determining unsuccessful cannulation events and failure to maturation in arteriovenous fistulas (REDUCE FTM I). J Am Soc Nephrol. 2006;17(11):3204-3212.

14. Dember LM, Imrey PB, Beck GJ, et al; Hemodialysis Fistula Maturation Study Group. Objectives and design of the hemodialysis fistula maturation study. Am J Kidney Dis. 2014;63(1):104-112.

15. Polo JR, Vázquez R, Polo J, Sanabia J, Rueda JA, Lopez-Baena JA. Brachiocephalic jump graft fistula: an alternative for dialysis use of elbow crease veins. Am J Kidney Dis. 1999;33(5):904-909.

16. Stehman-Breen CO, Sherrard DJ, Gillen D, Caps M. Determinants of type and timing of initial permanent hemodialysis vascular access. Kidney Int. 2000;57(2):639-645.

17. Applebaum H, Shashikumar VL, Somers LA, et al. Improved hemodialysis access in children. J Pediatr Surg. 1980;15:764-769.

18. Davidson I, Gallieni M, Saxena R, Dolmatch B. A patient centered decision making dialysis access algorithm. J Vasc Access. 2007;8: 59-68.

19. National Kidney Foundation. K/DOQI Clinical Practice Guidelines For Vascular Access, 2000. Am J Kidney Dis. 2001;37(Suppl 1): S137-S181.

20. Pisoni RL, Young EW, Dykstra DM, et al. Vascular access use in Europe and the United States: results from the DOPPS. Kidney Int. 2002;61:305-316

21. Sidawy AN, Gray R, Besarab A, et al. Recommended standards for report dealing with arteriovenous hemodialysis accesses. J Vasc Surg. 2002;35:603-610.

22. Ethier JH, Lindsay RM, Barre PE, Kappel JE, Carlisle EJ, Common A. Clinical practice guidelines for vascular access. Canadian Society of Nephrology. J Am Soc Nephrol. 1999;10(Suppl 13):S297-S305.
23. Golledge J, Smith CJ, Emery J, Farrington K, Thompson HH. Outcome of primary radiocephalic fistula for haemodialysis. Br J Surg. 1999;86:211-216.

24. Murphy GJ, White SA, Knight AJ, Doughman T, Nicholson ML. Longterm results of arteriovenous fistulas using transposed autologous basilic vein. Br J Surg. 2000;87:819-823.

25. Murphy GJ, White SA, Nicholson ML. Vascular access for haemodialysis. Br J Surg. 2000;87(10):1300-1315.

26. Robbin ML, Gallichio MH, Deierhoi MH, Young CJ, Weber TM, Allon M. US vascular mapping before hemodialysis access placement. Radiology. 2000;217:83-88.

27. Silva MB Jr, Hobson RW 2nd, Pappas PJ, et al. A strategy for increasing use of autogenous hemodialysis acces procedures: impact of preoperative noninvasive evaluation. J Vasc Surg. 1998;27:302-307.

28. Benedetto F, Carella G, Lentini S, et al. Use of bovine mesenteric vein in rescue vascular access surgery. J Vasc Access. 2010;11(2):112-114.

29. Khadra MH, Dwyer AJ, Thompson JF. Advantages of polytetrafluoroethylene arteriovenous loops in the thigh for hemodialysis access. Am J Surg. 1997;173:280-283.

30. Salimi J. Patency rate and complications of vascular access grafts for hemodialysis in lower extremities. Saudi J Kidney Dis Transpl. 2008;19:929-932.

31. Slater ND, Raftery AT. An evaluation of expanded polytetrafluoroethylene (PTFE) loop grafts in the thigh as vascular access for haemodialysis in patients with access problems. Ann R Coll Surg Engl. 1988;70:243-245.

32. Englesbe MJ,Al-Holou WN, Moyer AT, et al. Single centre review of femoral arteriovenous grafts for hemodialysis. World J Surg. 2006;30:171-175.

33. Rizzuti RP, Hale JC, Burkart TE. Extended patency of expanded polytetrafluoroethylene grafts for vascular access using optimal configuration and revisions. Surg Gynecol Obstet. 1988;166:23-27.

34. Roy-Chaudhury P, Kelly BS, Miller MA, et al. Venous neointimal hyperplasia in polytetrafluoroethylene dialysis grafts. Kidney Int. 2001;59:2325-2334.

35. May RE, Himmelfarb J, Yenicesu M, et al. Predictive measures of vascular access thrombosis: a prospective study. Kidney Int. 1997;52: 1656-1662.

36. Staalsen NH, Ulrich M, Winther J, Pedersen EM, How T, Nygaard H. The anastomosis angle does change the flow fields at vascular endto-side anastomoses in vivo. J Vasc Surg. 1995;21:460-471.

37. Kaufman JS. Antithrombotic agents and the prevention of access thrombosis. Semin Dial. 2000;13(1):40-46.

38. Lok CE, Moist L, Hemmelgarn BR, et al; Fish Oil Inhibition of Stenosis in Hemodialysis Grafts (FISH) Study Group. Effect of fish oil supplementation on graft patency and cardiovascular events among patients with new synthetic arteriovenous hemodialysis grafts: a randomized controlled trial. JAMA. 2012;307(17):1809-1816.

39. Taylor G, Gravel D, Johnston L, Embil J, Holton D, Paton S. Prospective surveillance for primary bloodstream infections occurring in Canadian hemodialysis units. Infect Control Hosp Epidemiol. 2002;23:716-720.

40. Kaplowitz LG, Comstock JA, Landwehr DM, Dalton HP, Mayhall CG. Prospective study of microbial colonization of the nose and skin and infection of the vascular access site in hemodialysis patients. J Clin Microbiol. 1988;26:1257-1262.

41. Ryan SV, Calligaro KD, Scharff J, Dougherty MJ. Management of infected prosthetic dialysis arteriovenous grafts. J Vasc Surg. 2004;39:73-78.

42. National Kidney Foundation, Inc. KDOQI. Guideline 27: Treatment do pseudoaneurysm of dialysis AV grafts (NKF KDOQI GUIDELINES 2000). New York: National Kidney Foundation, Inc; 2001. Available from: https://www.kidney.org/professionals/kdoqi/guidelines_updates/ doqiupva_iv.html\#17. Accessed June 5, 2014.

43. Shemesh D, Goldin I, Zaghal I, Berelowitz D, Verstandig AG, Olsha O. Stent graft treatment for hemodialysis access aneurysms. J Vasc Surg. 2011;54:1088-1094.

44. Shah AS, Valdes J, Charlton-Ouw KM, et al. Endovascular treatment of hemodialysis access pseudoaneurysms. JVasc Surg. 2012;55: 1058-1062. 
45. Bellinghieri G, Ricciardi B, Costantino G, et al. Exhaustion of vascular endowment in hemodialysis: proposal for a permanent inlet access. Int J Artif Organs. 1998;21(4):201-204.

46. National Institute for Clinical Excellence. Guidance on the use of ultrasound locating devices for placing central venous catheters. Technology Appraisal Guidance No 49. London: National Institute for Clinical Excellence; 2002. Available from: http://www.nice.org.uk/nicemedia/ pdf/Ultrasound_49_guidance.pdf. Accessed June 5, 2014.

47. Dolla D, Cavatorta F, Galli S, Zollo A, Ervo S. Anatomical variations of the internal jugular vein in non-uremic outpatients. J Vasc Access. 2001;2(2):60-63.

48. Lamperti M, Bodenham AR, Pittiruti M, et al. International evidence-based recommendations on ultrasound-guided vascular access. Intensive Care Med. 2012;38(7):1105-1117.

49. Karakitos D, Labropoulos N, De Groot E, et al. Real-time ultra-sound guided catheterisation of the internal jugular vein: a prospective comparison with the landmark technique in critical care patients. Crit Care. 2006;10(6):R162.

50. Clenaghan S, McLaughlin RE, Martyn C, McGovern S, Bowra J. Relationship between Trendelenburg tilt and internal jugular vein diameter. Emerg Med J. 2005;22:867-868.

51. Graham AS, Ozment C, Tegtmeyer K, Lai S, Braner DA. Videos in clinical medicine. Central venous catheterization. $N$ Engl J Med 2007;356(21):e21.

52. Khatri VP, Wagner-Sevy S, Espinosa MH, Fisher JB. The internal jugular vein maintains its regional anatomy and patency after carotid endarterectomy: a prospective study. Ann Surg. 2001;233: 282-286.

53. Rossi UG, Rigamonti $\mathrm{P}$, Tichà V, et al. Percutaneous ultrasound-guided central venous catheters: the lateral in-plane technique for internal jugular vein access. J Vasc Access. 2014;15(1):56-60.

54. Jankovic Z, Boon A, Prasad R. Fatal haemothorax following large-bore percutaneous cannulation before liver transplantation. Br J Anaesth. 2005;95(4):472-476.

55. Yoffa D. Supraclavicular subclavian venepuncture and catheterization. Lancet. 1965;2:614-617.

56. Hameeteman M, Bode AS, Peppelenbosch AG, van der Sande FM, Tordoir JH. Ultrasound-guided central venous catheter placement by surgical trainees: a safe procedure? Vasc Access. 2010;11(4): 288-292.

57. McGee DC, Gould MK. Preventing complications of central venous catheterization. $N$ Engl J Med. 2003;348(12):1123-1133.

58. Kusminsky RE. Complications of central venous catheterization. J Am Coll Surg. 2007;204(4):681-696.

59. Bishop L, Dougherty L, Bodenham A, et al. Guidelines on the insertion and management of central venous access devices in adults. Int J Lab Hematol. 2007;29(4):261-278.

60. Merrer J, De Jonghe B, Golliot F, et al. Complications of femoral and subclavian venous catheterization in critically ill patients. JAMA 2001;286:700-707.

61. Santoro D, Postorino A, Condemi CG, et al. Tunneled dialysis catheter and pacemaker leads determining superior vena cava syndrome. J Vasc Access. 2011;12(3):271-272.

62. Swindle MM, Nolan T, Jacobson A, Wolf P, Dalton MJ, Smith AC. Vascular access port (VAP) usage in large animal species. Contemp Top Lab Anim Sci. 2005;44(3):7-17.

63. Colas A, Curtis J. Silicone biomaterials - history and chemistry. In: Rutner BD, Hoffman AS, Schoen FJ, Lemons JE, editors. Biomaterials Science: an Introduction to Materials in Medicine. Boston: Elsevier Academic Press; 2004:80-86.

64. Colas A, Curtis J. Medical applications of silicones. In: Rutner BD, Hoffman AS, Schoen FJ, Lemons JE, editors. Biomaterials Science: an Introduction to Materials in Medicine. Boston: Elsevier Academic Press; 2004:697-707.

65. Brown JM. Polyurethane and silicone: myths and misconceptions. J Intraven Nurs. 1995;18(3):120-122.
66. Cohen AB, Dagli M, Stavropoulos SW Jr, et al. Silicone and polyurethane tunneled infusion catheters: a comparison of durability and breakage rates. J Vasc Interv Radiol. 2011;22(5):638-641.

67. Savader S, Trerotola S, editors. Venous Interventional Radiology with Clinical Perspectives. 2nd edition. New York: Thieme Medical Publisher, Inc.; 2000.

68. Rupp ME, Lisco SJ, Lipsett PA, et al. Effect of a second-generation venous catheter impregnated with chlorhexidine and silver sulfadiazine on central catheter-related infections: a randomized, controlled trial. Ann Intern Med. 2005;143(8):570-580.

69. Maki DG, Stolz SM, Wheeler S, Mermel LA. Prevention of central venous catheter-related bloodstream infection with an antisepticimpregnated catheter: a randomized, controlled trial. Ann Intern Med. 1997; 127(4):257-266.

70. McCann M, Moore ZEH. Interventions for preventing infectious complications in haemodialysis patients with central venous catheters. Cochrane Database Syst Rev. 2010;(1):CD006894.

71. Astor BC, Eustace JA, Powe NR, Klag MJ, Fink NE, Coresh J; CHOICE Study. Type of vascular access and survival among incident hemodialysis patients: the Choices for Healthy Outcomes in Caring for ESRD (CHOICE) Study. J Am Soc Nephrol. 2005;16(5): 1449-1455.

72. Mermel LA, Allon M, Bouza E, et al. Clinical practice guidelines for the diagnosis and management of intravascular catheter-related infection: 2009 update by the Infectious Diseases Society of America. Clin Infect Dis. 2009;49(1):1-45.

73. Engemann JJ, Friedman JY, Reed SD, et al. Clinical outcomes and cost due to Staphylococcus aureus bacteremia among patients receiving long-term hemodialysis. Infect Control Hosp Epidemiol. 2005;26(6): 534-539.

74. Maki DG, Kluger DM, Crinich CJ. The risk of bloodstream infection in adults with different intravascular devices: a systematic review of 200 published prospective studies. Mayo Clin Proc. 2006;81(9): 1159-1171.

75. CDC (Centers for Disease Control and Prevention). Vital signs: central line-associated bloodstream infections - United States, 2001, 2008, and 2009. MMWR Morb Mortal Wkly Rep. 2011;60:243-248.

76. Crnich CJ, Maki DG. The promise of novel technology for the prevention of intravascular device-related bloodstream infection. I. Pathogenesis and short-term devices. Clin Infect Dis. 2002;34(9): 1232-1242.

77. Wisplinghoff H, Bischoff T, Tallent SM, Seifert H, Wenzel RP, Edmond MB. Nosocomial bloodstream infections in US hospitals: analysis of 24,179 cases from a prospective nationwide surveillance study. Clin Infect Dis. 2004;39(3):309-317.

78. Safdar N, Fine JP, Maki DG. Meta-analysis: methods for diagnosing intravascular device-related bloodstream infection. Ann Intern Med. 2005;142(6):451-466

79. Blot F, Schmidt E, Nitenberg G, et al. Earlier positivity of centralvenous- versus peripheral-blood cultures is highly predictive of catheter-related sepsis. J Clin Microbiol. 1998;36(1):105-109.

80. Allon M. Treatment guidelines for dialysis catheter-related bacteremia: an update. Am J Kidney Dis. 2009;54(1):13-17.

81. National Institute for Health and Care Excellence. CG139: Infection: prevention and control of healthcare-associated infections in primary and community care. Available from: http://publications.nice.org. uk/infection-cg139/other-versions-of-this-guideline\#full-guideline. Accessed May 3, 2014.

82. Vanholder R, Canaud B, Fluck R, et al. Diagnosis, prevention and treatment of haemodialysis catheter-related blood stream infections (CRBSI): a position statement of European Renal Best Practice (ERBP). NDT Plus. 2010;3(3):234-246.

83. Messing B, Peitra-Cohen S, Debure A, Beliah M, Bernier JJ. Antibioticlock technique: a new approach to optimal therapy for catheter-related sepsis in home parenteral nutrition patients. JPEN J Parenter Enteral Nutr. 1988;12(2):185-189. 
84. CDC.gov [homepage on the Internet]. CDC Approach to BSI Prevention in Dialysis Facilities. Available from: http:/www.cdc.gov/ dialysis/PDFs/Dialysis-Core-Interventions-5_10_13.pdf. Accessed December 8, 2013.

85. Milburn JA, Cassar K, Ford I, Fluck N, Brittenden J. Prothrombotic changes in platelet, endothelial and coagulation function following hemodialysis. Int J Artif Organs. 2011;34(3):280-287.

86. Milburn JA, Ford I, Mutch NJ, Fluck N, Brittenden J. Thrombin-anti-thrombin levels and patency of arterio-venous fistula in patients undergoing haemodialysis compared to healthy volunteers: a prospective analysis. PLoS One. 2013;8(7):e67799.

87. NKF-KDOQI. Clinical practice guidelines for vascular access. Am J Kidney Dis. 2006;48(Suppl 1):S248-S272.
88. Tordoir J, Canaud B, Haage P, et al. European best practice guidelines (EBPG) on vascular access. Nephrol Dial Transplant. 2007; 22(Suppl 2):ii88-ii117.

89. Ravani P, Palmer SC, Oliver MJ, et al. Associations between hemodialysis access type and clinical outcomes: a systematic review. J Am Soc Nephrol. 2013;24:465-473.

90. Grubbs V, Wasse H, Vittinghoff E, et al. Health status as a potential mediator of the association between hemodialysis vascular access and mortality. Nephrol Dial Transplant. 2014;29:892-898.

\section{Publish your work in this journal}

The International Journal of Nephrology and Renovascular Disease is an international, peer-reviewed open-access journal focusing on the pathophysiology of the kidney and vascular supply. Epidemiology, screening, diagnosis, and treatment interventions are covered as well as basic science, biochemical and immunological studies. The journal welcomes original research, clinical studies, reviews \& evaluations, expert opinion and commentary, case reports and extended reports. The manuscript management system is completely online and includes a very quick and fair peerreview system, which is all easy to use. Visit http://www.dovepress.com/ testimonials.php to read real quotes from published authors.

Submit your manuscript here: http://www.dovepress.com/international-journal-of-nephrology-and-renovascular-disease-journal 